\title{
REVIEW
}

\section{Revisited role for mucus hypersecretion in the pathogenesis of COPD}

\author{
I. Cerveri* and V. Brusasco"\#
}

ABSTRACT: Chronic obstructive pulmonary disease (COPD) is a heterogeneous and complex disease of which the basic pathophysiological mechanisms remain largely unknown. On the basis of recent results from pathological studies and large clinical trials, the presence of airway inflammation does not seem to be sufficient to explain the complexity of the disease and the relatively poor response to treatment. It is probably time to abandon the concept of COPD as a unique disease and define, identify and treat the various aspects, which may differ between individuals.

Among the different phenotypic distinctions, the classical distinction "chronic bronchitis" has mucus hypersecretion as the key presenting symptom. Its role in COPD has been the subject of an ongoing debate; however, it now appears to be being re-evaluated due to findings from recent epidemiological and pathological studies. In this context, the view that chronic mucus hypersecretion plays a secondary role in the pathogenesis of COPD should be abandoned and instead, drugs targeting mucus hypersecretion should be considered as a treatment option.

\section{KEYWORDS: Chronic obstructive pulmonary disease, mucus hypersecretion}

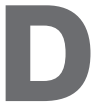
espite the fact that extensive resources have been invested in research on the pathogenesis and treatment of chronic obstructive pulmonary disease (COPD) in the past 20 yrs, COPD remains a major public-health problem and is progressing up the list of priorities for major health organisations, such as the World Health Organization [1]. In fact, COPD worsens the quality of life of patients, increases their contact with healthcare providers, is responsible of a large proportion of hospital admissions for acute care, and carries an increased risk of disability and premature death.

Several epidemiological studies have well documented that long-term exposure to cigarette smoke or environmental pollution, or both, are the key risk factors for COPD. This knowledge certainly makes COPD a preventable disease, but the relatively poor knowledge of its pathophysiology makes it a difficult disease to treat, particularly in the early stages. Recent pathological findings, in conjunction with the scarce benefits of anti-inflammatory treatments reported in recent large clinical trials, strongly suggest that the presence of airway inflammation may not be enough to explain the distinct pathophysiological processes and treatment responses [2]. In a recent study it has been proposed that it is now time to abandon the concept of COPD as a unique disease and to define, identify and treat the different disorders that contribute to the pathogenesis of such a complex condition [3]. In this context, the old view that chronic mucus hypersecretion plays a secondary role in the pathogenesis of COPD should be re-evaluated [4, 5], together with the use of drugs targeting mucus hypersecretion.

\section{COPD AS A HETEROGENEOUS DISEASE}

The classical approach to COPD has encompassed different disorders in a unique entity under a variety of imprecise definitions and diagnostic criteria. The current definition of COPD is based on the presence of airflow obstruction that is not completely reversible with inhaled bronchodilators, irrespective of the presence of chronic cough and sputum or the existence of emphysematous changes in the lungs [6]. It fails to take into account the heterogeneity of the disease and does not reflect the different phenotypic distinctions, which may be associated with differing natural histories, clinical features and response to treatments. A major practical problem with disregarding the heterogeneity of COPD is that not all individual patients may respond to a given

\section{AFFILIATIONS}

${ }^{*}$ Clinica di Malattie dell'Apparato Respiratorio, Fondazione Ricovero e Cura a Carattere Scientifico

Policlinico S. Matteo, Università di Pavia, Pavia, and

"Dipartimento di Medicina Interna, Università di Genova, Genova, Italy.

\section{CORRESPONDENCE}

I. Cerveri

Clinica Malattie dell'Apparato

Respiratorio

Fondazione IRCCS Policlinico San Matteo

Via Taramelli

27100 Pavia

Italy

E-mail: icerveri@smatteo.pv.it

Received:

March 162010

Accepted after revision:

March 292010

\section{PROVENANCE}

Publication of this peer-reviewed article was supported by Dompé SPA, Italy (unrestricted grant, European Respiratory Review issue 116).

European Respiratory Review Print ISSN 0905-9180 Online ISSN 1600-0617 
treatment and, therefore, the opportunity to optimise therapy may be missed. Recent studies have identified different clusters of COPD patients based on both clinical and physiological characteristics. These include a high frequency of exacerbations, rapid decline of lung function, airway hyperresponsiveness, impaired exercise tolerance and emphysema versus airways disease [7, 8]. This large heterogeneity of COPD phenotypic distinctions may explain the failure of some large clinical trials to achieve statistically significant effects on primary outcomes.

\section{THE CURRENT PATHOGENETIC THEORY AND ITS LIMITS}

There are a plethora of hypotheses relating COPD to specific pathogenetic culprits, but the basic mechanisms remain largely unknown as no single mechanism can account for the complex pathology in COPD. It is likely that interactions occur among different mechanisms, such as airway inflammation, protease/ antiprotease imbalance, oxidative stress and apoptosis, as destructive processes in emphysema. The traditional pathogenetic theory is based on the hypothesis that COPD is sustained by a perpetuating "burning" process mediated by inflammatory cells, pro-inflammatory cytokines and abnormally high oxidative stress. To confront the noxious gases and particles, the respiratory system reacts with the innate and adaptive inflammatory immune response mostly at the level of its smallest and most delicate airways. The inflammatory cell infiltration into lung tissue in COPD is inextricably linked to abnormal tissue repair and the remodelling process that increases the number and size of bronchial mucus glands, the mucus content of airway epithelium, the proliferative activity of the epithelial cells and causes squamous cell metaplasia. Inflammatory exudates containing mucus occlude the airway lumen, and a tissue-and-repair process thickens the walls, thus narrowing the conducting airways to $<2 \mathrm{~mm}$ in diameter (fig. 1). A multivariate analysis of this histology indicates that thickening of the walls of small conducting airways and occlusion of the lumen by inflammatory exudates containing mucus can explain more of the variance in the association between forced expiratory volume in $1 \mathrm{~s}$ (FEV1) decline and histology than the infiltration by any inflammatory cell type. Emphysematous destruction of gas-exchanging tissue also contributes to airflow obstruction by decreasing the elastic recoil pressure available to drive air out of the lung during expiration. This tissue destruction begins in the respiratory bronchioles in very close proximity to the small conducting airways, which become the major site of obstruction in COPD. The precise mechanisms causing small airways to thicken and narrow in such close proximity of lung tissue undergoing emphysematous destruction remains to be detected [9]. The accumulation of tissue responsible for thickening the small conducting airways, called "exaggerated tissue repair", is a very different process to the emphysematous destruction of the gas-exchanging tissue, called "insufficient tissue repair" $[10,11]$.

The hypothesis that inflammation accounts for all features of the pathobiological processes involved in alveolar destruction and airway remodelling in COPD oversimplifies the overall polymorphic and multi-factorial nature of inflammation in COPD and, consequently, has not led to major therapeutic advances in the treatment of COPD. Several observations, particularly related to the progressive nature of COPD, raise some questions that have not been adequately addressed such as: Why does it take decades for the disease to develop? Why do some patients experience a rapid decline in lung function while others live for a long time with stable airflow obstruction? Why does inflammation persist despite smoking cessation? Why do corticosteroids have little impact on the natural history of COPD, except for exacerbations?

\section{"THE BRITISH HYPOTHESIS" AND CHRONIC MUCUS HYPERSECRETION}

In the classical phenotype of chronic bronchitis, mucus hypersecretion is the key presenting symptom and its contribution to airflow obstruction and has been the subject of debate for a long time [12]. By the 1950s it was known that tobacco smoking was associated with chronic cough and sputum production, and that smokers who developed chronic bronchitis had impaired lung defences, a condition favouring colonisation and infection of the lower airways [13]. Post mortem examinations of lungs showed that the excess mucus production which defined chronic bronchitis was associated with enlarged bronchial glands and that an approximate relationship existed between the presence of chronic bronchitis and emphysema [14].

A concept that subsequently became known as "the British hypothesis" proposed that smoking caused mucus hypersecretion and impaired host defences leading to chronic infection, disseminated bronchiolar obstruction and emphysema. This hypothesis was eventually rejected due to the findings of a seminal longitudinal study of working males from West London (UK), conducted from 1961 to 1969, where only a fraction of smokers developed COPD; this was independent of the absence or presence of chronic bronchitis [15]. As an alternative explanation it was, therefore, proposed that
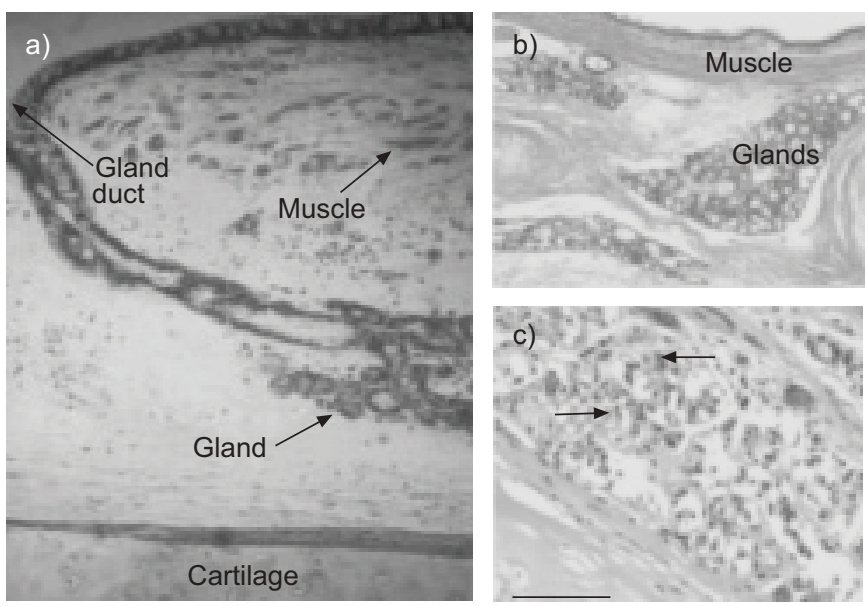

FIGURE 1. Pathological changes of the central airways in chronic obstructive pulmonary disease. a) A central bronchus from the lung of a cigarette smoker with normal pulmonary function. Only small amounts of bronchial smooth muscle are present and the epithelial glands are small. b) In contrast, a subject with chronic bronchitis has bronchial smooth muscle that appears as a thick bundle and enlarged bronchial glands. c) The enlarged bronchial glands at a higher magnification. A chronic inflammatory process involving polymorphonuclear leukocytes (arrowhead) and mononuclear cells, including plasma cells (arrows) can also be seen. Reproduced from [11], with permission from the publisher. 
ECRHS I

(1991-1993)
ECRHS II

(1998-2002)

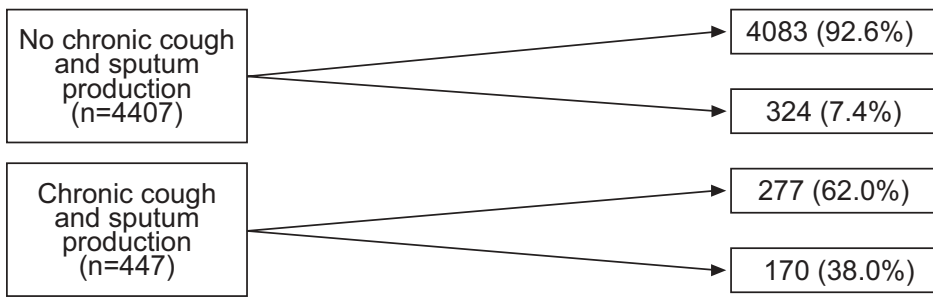

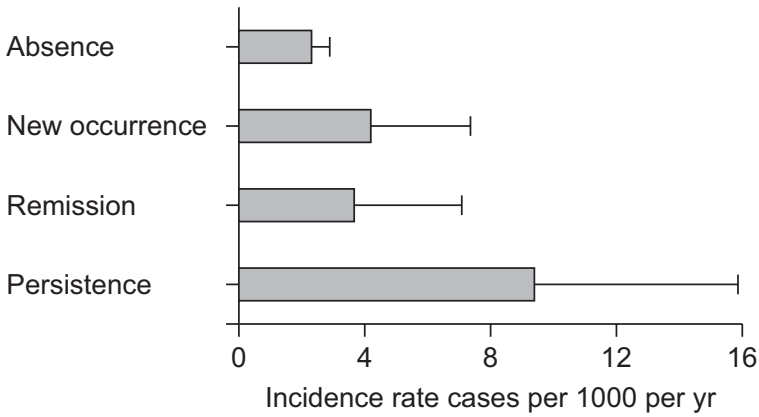

FIGURE 2. Incidence of chronic obstructive pulmonary disease according to the history of chronic cough/phlegm. The statistically significant incidence rate ratio (persistence versus absence) was $4.04(95 \% \mathrm{Cl}$ 2.29-7.11). ECRHS: European Community Respiratory Health Survey. Reproduced from [23] with permission from the publisher.

"emphysema and intrinsic disease of small airways were involved to different degrees in these obstructed men" [15] and that the symptoms of chronic mucus hypersecretion in the absence of airflow obstruction was a benign condition that did not progress into COPD. Consequently, clinicians have long relegated mucus to the medical dustbin of annoying yet harmless symptoms.

However, results of several recent epidemiological studies showing an association between mucus hypersecretion and outcomes in patients with COPD have re-ignited the old controversy and opinions are changing rapidly. In a 22-yr mortality survey of 1,061 working males, respiratory mucus hypersecretion was found to constitute more than a negligible public health problem [16]. In the famous study on COPD mortality in six US cities, respiratory symptoms remained a significant predictor of mortality after adjusting for the level of lung function [17]. In the Copenhagen City Heart Study, chronic mucus hypersecretion was found to be significantly and consistently associated with both an excess in FEV1 decline

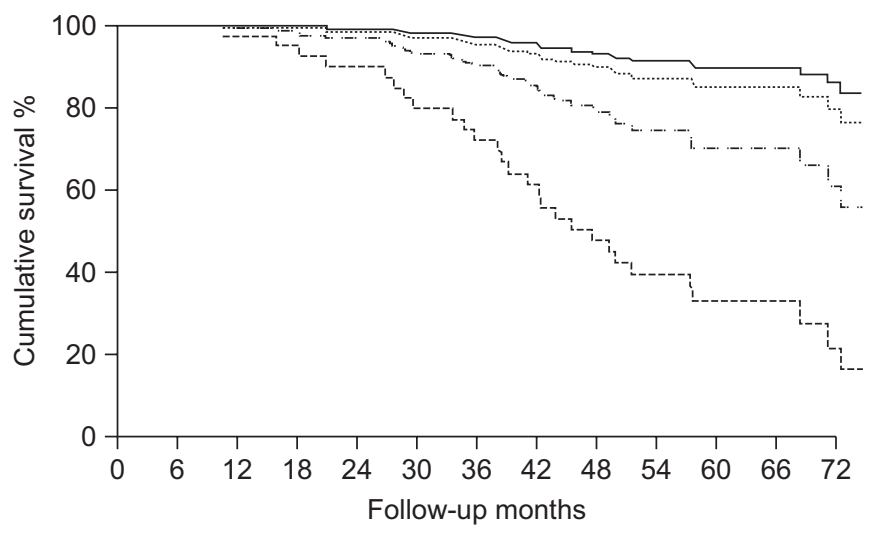

FIGURE 3. Kaplan-Meier survival plot of 101 cases of severe (Global Initiative for Chronic Obstructive Lung Disease (GOLD) stage 3) and very severe (GOLD stage 4) chronic obstructive pulmonary disease following lung volume reduction surgery. The median survival was shortened in the quartile with the most severe occlusion by mucous exudate on the fully expanded lumen ( $\mathrm{HR} 3.28,95 \% \mathrm{Cl} 1.55-$ 6.92; $p=0.002)$ after adjustment for several factors. —_: quartile 1 (smallest) …....: quartile 2 ; -...-: quartile 3 ; - - - -: quartile 4 (largest). Reproduced from [25] with permission from the publisher. and an increase of subsequent hospitalisations due to COPD [18]. An article published in 2002 by MELTON [19] resumed this topic. This topic has subsequently been the subject of a Novartis Foundation symposium, which quoted these epidemiological studies and concluded that dealing with the inflammation in COPD patients might be not be sufficient, and that clearing mucus from the airways might provide a better opportunity to treat the inflammation and offer relief to the patients [20]. In 2001, a new classification of stage 0 (patients who present with chronic cough and phlegm with normal lung function considered "at risk" for the development of COPD) was added to the Global Initiative for Chronic Obstructive Lung Disease classification guidelines but was subsequently removed in the 2006 updated guidelines because of controversy regarding its real predictive value [21, 22]. To test whether chronic cough and phlegm are independent predictors of COPD, the database of an international cohort of $>5,000$ subjects without asthma (aged 20-44 yrs) with normal lung function from 12 countries followed from 1991 to 2002 as part of the European Community Respiratory Health Survey was re-analysed [23]. The incidence of COPD in young adults who confirmed the presence of persistent chronic cough and phlegm at the end of follow-up was three-fold higher than the incidence in subjects who had never reported these symptoms, independent of their smoking habits (fig. 2) [23]. The conclusions of this large European follow-up study have recently been reinforced and extended by an analysis of the Framingham Offspring cohort (participants aged 13-80 yrs) regularly followed for $\leqslant 26$ yrs [24]. This analysis has offered the natural history of chronic airflow obstruction to be revisited showing that the presence of chronic cough and phlegm at baseline or the diagnosis of a respiratory disorder during follow-up appears to identify a subpopulation of smokers who are particularly susceptible to the deleterious effects of smoking on their lungs. The authors claim that this novel finding has important clinical relevance [24]. Another study from HogG et al. [25] demonstrates the results of the relationship between small airways pathology and long-term mortality in 101 patients with COPD who had undergone lung volume reduction surgery. Interestingly, in this pathology study the only parameter significantly related to survival was the severity of small airway occlusion by exudates containing mucus, but not by other indexes of airway inflammation (fig. 3) [25]. 
Even if mucus production appears to be particularly important in COPD, its precise role in the development and progression of the disease is not completely understood. HoGG et al. [25] have proposed that mucus could increase in the small airways, either because it is produced in excess by goblet cells stimulated by neuthophil elastase or oxygen free-radicals, or because it is unable to flow out as a result of impaired mucus clearance. The mucus could also stick to airways walls, thus contributing to luminal occlusion and lung function decline because of its viscosity. In a recent review on the role of airway epithelial stem cells in the pathophysiology of COPD, it has been hypothesised that the initial event may be the differentiation of airway stem cells as part of an injury-repair process [26].

\section{CONCLUSIONS}

Altogether, the most recent findings of epidemiological and pathological studies have led to a new hypothesis that airway inflammation and mucus production in COPD may be somewhat dissociated. Therefore, in the new challenges to elucidate the pathophysiology of COPD and to define the different phenotypic distinctions that can be useful in the choice of treatment in individual patients, more attention should be paid to the role of chronic mucus hypersecretion, particularly in the early stages of COPD.

\section{STATEMENT OF INTEREST}

I. Cerveri has received funds for research from Boehringer Ingelheim $(€ 15,000)$, Pfizer $(€ 15,000)$ and Novartis $(€ 15,000)$. I. Cerveri has also received fees for consultancy from AstraZeneca $(€ 10,000)$ and Dompe (€6,000). V. Brusasco received $€ 6,250$ in 2005 and $€ 3,800$ in 2006 for serving on an advisory board for Altana; $€ 3000$ in 2005 and $€ 3,500$ in 2008 from Lofarma for consultancies; $€ 1,500$ in 2005, $€ 8,325$ in 2006, $€ 2,500$ in 2007 and $€ 6,600$ in 2008 for speaking at conferences sponsored by Boehringer Ingelheim; $€ 2,200$ from Boehringer Ingelheim for consultancy in 2009; $\$ 1,500$ from Deep Breeze for consultancy in 2009; €6,000 from Dompé for consultancy in 2009; £960 in 2006 and $£ 1,200$ in 2008 from GlaxoSmithKline for participating in advisory boards; $€ 6,000$ in 2006 and $€ 3000$ in 2007 from Almirall for serving on an advisory board; $€ 3,000$ in 2006 from Forest Laboratories for serving on an advisory board; and $€ 1,500$ from Menarini for consultancy in 2008. V. Brusasco's institution received $€ 20,000$ in 2006 as an unrestricted research grant from Boehringer Ingelheim, and participated in several multicentre clinical trials financed by pharmaceutical companies (Chiesi, MSD, Boehringer Ingelheim, GlaxoSmithKline and Novartis).

\section{REFERENCES}

1 World Health Organization. 2008-2013 Action Plan for the Global Strategy for the Prevention and Control of Noncommunicable Diseases. 2008. Available from: www.who.int/nmh/publications / ncd_action_plan_en.pdf

2 Brusasco V, Crimi E, Pellegrino R. Airway inflammation in COPD: friend or foe? Am I Respir Crit Care Med 2007; 176: 425-426.

3 Beasley R, Weatherall M, Travers J, et al. Time to define the disorders of the syndrome of COPD. Lancet 2009; 374: 670-672.

4 Wedzicha JA, Seemungal TAR. COPD exacerbations: defining their cause and prevention. Lancet 2007; 370: 786-796.

5 Burgel PR, Nesme-Meyer P, Chanez P, et al. Cough and sputum production are associated with frequent exacerbations and hospitalizations in COPD subjects. Chest 2009; 135: 975-982.
6 Global Strategy for the Diagnosis, Management and Prevention of COPD. Global Initiative for Chronic Obstructive Lung Disease. 2008. www.goldcopd.org Date last updated: January 2009. Date last accessed: July 2, 2009.

7 Friedlander AL, Lynch D, Dyar LA, et al. Phenotypes of chronic obstructive pulmonary disease. COPD 2007; 4: 355-384.

8 Weatherall M, Travers J, Shirtcliffe PM, et al. Distinct clinical phenotypes of airways disease defined by cluster analysis. Eur Respir J 2009; 34: 812-818.

9 Araya J, Cambier S, Markovics JA, et al. Squamous metaplasia amplifies pathologic epithelial-mesenchymal interactions in COPD patients. J Clin Invest 2007; 117: 3551-3562.

10 MacNee W. Pathogenesis of chronic obstructive pulmonary disease. Clin Chest Med 2007; 28: 479-513.

11 Hogg JC, Timens W. The pathology of chronic obstructive pulmonary disease. Annu Rev Pathol 2009; 4: 435-459.

12 Vesbo J, Hogg JC. Convergence of the epidemiology and pathology of COPD. Thorax 2006; 61: 86-88.

13 Brumfitt W, Willoghby MLN, Bromley LL. An evaluation of sputum examination in chronic bronchitis. Lancet 1957; 273: 1306-1309.

14 Thurlbeck WM, Angus GE. The relationship between chronic bronchitis and emphysema as assessed morphologically. Am Rev Respir Dis 1963; 87: 815-819.

15 Fletcher CM, Thinker CM. Chronic bronchitis. A further study of simple diagnostic methods in a working population. BMJ 1960; 1 : 1941-1948.

16 Annesi I, Kauffman F. Is respiratory mucus hypersecretion really an innocent disorder? A 22-year mortality survey of 1,061 working men. Am Rev Respir Dis 1986; 134: 688-693.

17 Speizer FE, Fay ME, Dockery DW, et al. Chronic obstructive pulmonary disease mortality in six U.S. cities. Am Rev Respir Dis 1989; 140: Suppl. 3, S49-S55.

18 Vestbo J, Prescott E, Lange P. Association of chronic mucus hypersecretion with FEV1 decline and chronic obstructive pulmonary disease morbidity. Copenhagen City Heart Study Group. Am J Crit Care Med 1996; 153: 1530-1535.

19 Melton L. Does mucus hypersecretion matter in airway disease? Lancet 2002; 359: 1924.

20 Vestbo J, Epidemiological studies in mucus hypersecretion. In: Chadwick DJ, Goode JA, eds. Novartis Foundation Symposium 248: Mucus Hypersecration in Respiratory Disease. Malden, Wiley 2002; pp. 277-282.

21 Vestbo J, Lange P. Can GOLD Stage 0 provide information of prognostic value in COPD? Am J Respir Crit Care Med 2002; 166: 329-332.

22 Mannino DM. GOLD stage 0 COPD. It is real? Does it matter? Chest 2006; 130: 2309-2310.

23 De Marco R, Accordini S, Cerveri I, et al. Incidence of chronic obstructive pulmonary disease in a cohort of young adults according to the presence of chronic cough and phlegm. Am J Respir Crit Care Med 2007; 175: 32-39.

24 Kohansal R, Martinez- Camblor P, Agusti A, et al. The natural history of chronic air flow obstruction revisited: an analysis of the Framingham offspring cohort. Am J Respir Crit Care Med 2009; 180: 3-10.

25 Hogg JC, Chu FS, Tan WC, et al. Survival after lung volume reduction in chronic obstructive pulmonary disease: insights from small airways pathology. Am J Respir Crit Care Med 2007; 176: 454-459.

26 Randell SH. Airway epithelial stem cells and the pathophysiology of COPD. Proc Am Thorac Soc 2006; 3: 718-725. 